\title{
Hypoxia-induced pulmonary vascular remodeling: a model for what human disease?
}

\author{
Norbert F. Voelkel and Rubin M. Tuder \\ Pulmonary Hypertension Center, University of Colorado Health Sciences Center, Denver, Colorado, USA \\ Address correspondence to: Norbert F. Voelkel, Division of Pulmonary Sciences and Critical Care Medicine, \\ University of Colorado Health Science Center, 4200 East Ninth Avenue, C272, Denver, Colorado 80262, USA. \\ Phone: (303) 315-4211; Fax: (303) 315-5632; E-mail: norbert.voelkel@uchsc.edu.
}

Exposure of most animals to low levels of environmental oxygen results in alveolar hypoxia and reliably causes chronic pulmonary hypertension and morphological alterations of the precapillary pulmonary vessels (1-4). Chronic hypoxic exposure of animals has been used for decades to induce pulmonary vascular remodeling and, more recently, to assess the roles in this process of lipid mediators (5), endothelin (6), serotonin (7), nitric oxide (NO) $(8,9)$, and prostacyclin $(10)$, as well as VEGF and its receptors $(11,12)$. As one measure of the clinical importance of work in this system, trials are now ongoing to test endothelin receptor blockers in the treatment of severe pulmonary hypertension.

Hypoxia, pulmonary vasoconstriction, and vascular remodeling

Chronic hypoxia can be induced by exposing animals to normal air at hypobaric pressures or to oxygen-poor air at normal pressure. In many but not all species, such treatments lead to muscularization of a subset of arterioles in the lung, the so-called precapillary "resistance vessels," which are particularly sensitive to the effects of hypoxia. Both vascular smooth muscle cells (VSMCs) and adventitial fibroblasts $(3,4)$ proliferate under these conditions, but no significant endothelial cell proliferation occurs. This is fortunate, since such proliferation would block the lumen of the arteriole and interfere with blood flow, as indeed occurs under conditions of severe pulmonary hypertension (see below). The muscularization of the precapillary pulmonary arterioles in response to chronic hypoxia and vasoconstriction is slowly reversible when normal oxygen levels are restored and may represent an adaptive response to elevated pulmonary artery pressure.

Pulmonary arterial muscularization is not specific for hypoxia. Increased pulmonary blood flow and the associated elevation in vascular shear stress can also produce muscularization, as can treatment with the plant alkaloid monocrotaline (13). The most commonly cited explanation for the precapillary vascular remodeling in the chronically hypoxic/hypoxemic lung is that hypoxia causes resistance vessel vasoconstriction, perhaps mediated by the closure of $\mathrm{K}^{+}$channels in precap- illary VSMCs $(14,15)$. Vasoconstriction leads to increased shear stress, which then, by analogy to these other conditions, is presumed to trigger hypertrophy and proliferation of VSMCs. The decrease in oxygen concentration in the alveoli surrounding the resistance vessels must therefore somehow be sensed by neighboring VSMCs. The events leading from a reduction in oxygen concentrations in the alveolar space to precapillary VSMC constriction are complex and incompletely understood. A more complete treatment of the vast literature of hypoxic vasoconstriction and its mechanisms can be found elsewhere (16).

\section{The complex pulmonary response}

to chronic hypoxia

Although precapillary vasoconstriction may well contribute to pulmonary arterial muscularization, it is doubtful that it triggers solely this response. Indeed, some of the vasoconstrictor substances released in the hypoxic lung tissue - endothelin and serotonin, in particular $(6,7)$ - serve as growth factors for VSMCs, independent of their effects on vascular tone. Moreover, the reduction in oxygen simultaneously activates a variety of cellular, biochemical, and molecular processes and multiple signals that converge on the vascular cells, and not all of these effects appear to act downstream of vasoconstriction. Some of the molecular and cellular events that may contribute to the structural changes in the vasculature of the lung are shown in Figure 1.

Transcriptional regulation during the response to hypoxia is crucial for vascular remodeling and involves several classes of signals and numerous target genes. Thus, promoters of some growth factor and other genes, including those for TGF- $\beta$, PDGF, and ICAM1 , contain shear stress response elements that are believed to mediate the induction of these genes during chronic hypoxia. Other factors, such as VEGF and erythropoietin, are induced directly by the transcription factor hypoxia-inducible factor $1 \alpha$ (HIF-1 $\alpha$ ) (refs. 17-19; see also Isner [ref. 20] and Semenza [ref. 21], this Perspective series). Still other genes may be controlled by increased production of reactive oxygen 


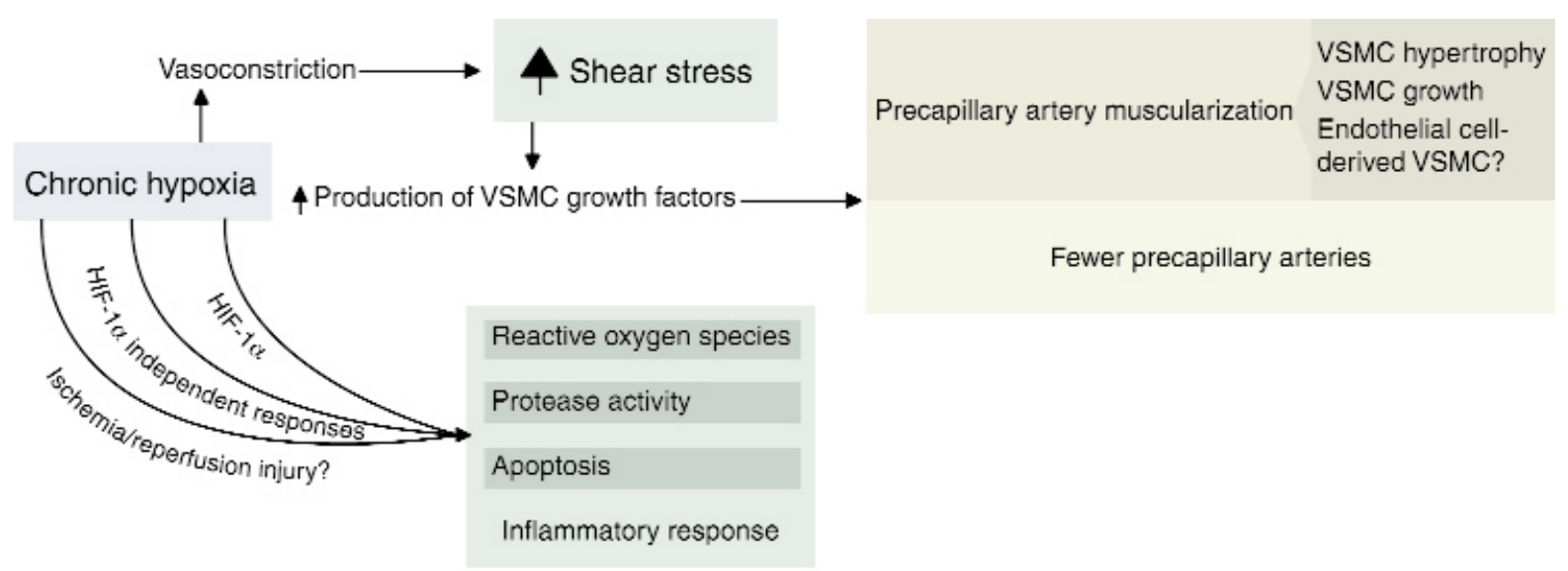

Figure 1

Mechanisms that may be involved in hypoxia-induced pulmonary vascular remodeling. Growth factors for VSMCs can be generated as consequences of elevated shear stress or of oxidant stress and may be dependent or independent of hypoxia-inducible factor $1 \alpha(\mathrm{HIF}-1 \alpha)$. ROSs may also affect gene expression, activate proteinases, and trigger the production of cytokines.

species (ROSs; ref. 22); because of regional decreases in blood flow, chronic hypoxic vasoconstriction can lead to ischemia/reperfusion injury, inducing inflammation and causing the accumulation of ROSs in the lung parenchyma (23). Such injury may be restricted to the initial stage of chronic hypoxia, or occur in situations of intermittent hypoxia/hypoxemia, as seen in the sleep apnea syndrome. Sources of ROSs in the hypoxemic lung include endothelium-derived xanthine-oxidase, cyclooxygenases, lipoxygenases, endothelial NO synthase (eNOS), VSMC-derived NADH oxidase, and activated macrophages. In aggregate, ROSs may deplete lung tissue NO and activate both cell proliferation and cell death (24).

The complex and multifactorial response to this single physicochemical stimulus, chronic hypoxia, makes it difficult to dissect the effects of potentially useful therapeutic agents. Thus, calcium-entry blockers are believed to prevent development of chronic hypoxiainduced pulmonary hypertension by inhibiting hypoxic vasoconstriction (25), but other drug actions are possible. Chronic treatment of rats with a platelet-activating factor antagonist, which does not affect pulmonary vascular constriction, decreases chronic hypoxia-induced pulmonary vascular remodeling and decreases the pulmonary hypertension (26). It should be noted that the increase in pulmonary artery pressure seen during acute hypoxic exposure results from vasoconstriction, whereas the pulmonary hypertension following chronic hypoxia can be explained to a large extent by the structural alteration of the small pulmonary arteries and may occur even in the absence of vasoconstriction. Thus, treatment of rats with anti-VEGF antibodies may not directly induce vasoconstriction, either acutely or chronically, but long-term exposure to this antibody enhances hypox- ia-induced pulmonary hypertension (27), associated with loss of pulmonary vasodilation and also increased vascular remodeling, likely because of the decreased production of prostacyclin (28) and NO (29).

\section{Expression of oxygen-sensitive genes in the lung} Recently, Yu and colleagues (30) reported impaired pulmonary vascular remodeling in mice carrying only a single functional copy of the HIF-1 $\alpha$ gene. HIF- $1 \alpha$ is critical for the upregulation of VEGF (19) and the subsequent increase in $\mathrm{NO}$ and prostacyclin production $(28,29)$ during hypoxic episodes. Because these latter molecules strongly suppress vascular remodeling, it might have been anticipated that $H I F-1 \alpha^{+/-}$animals would develop more pulmonary vascular remodeling than wild-type controls. Since this is, surprisingly, not the case, it appears that other HIF-1 $\alpha$ target genes, whose products promote pulmonary vascular remodeling, are also weakly expressed in the heterozygotes. One intriguing possibility is that impaired expression of glucose transporters and glycolytic enzymes (31) suppresses vascular remodeling responses in these animals, much as starvation does.

The vascular remodeling response to chronic hypoxia likely involves extravascular cells, such as alveolar macrophages and alveolar type II cells. Alveolar type II cells are known to synthesize prostacyclin (32), and Geraci et al. (10) showed recently that overexpression of prostacyclin synthase in these cells prevents the development of hypoxic pulmonary hypertension in mice. Hypoxic alveolar macrophages upregulate expression of the transcription factor Egr-1 (33), which activates the de novo synthesis of tissue factor, thus generating a procoagulant environment in the hypoxic lung. Thus, homozygous Egr1-null mice exposed to acute hypoxia do not show either enhanced tissue factor 
expression or pulmonary vascular fibrin deposition (33). Recently, David Stern's group demonstrated that hypoxia leads to expression of the Egr-1 protein not only in phagocytes, but also in VSMCs (33), and they found that $P K C \beta$ knockout mice are unable to induce Egr-1 in response to hypoxia (34). Interestingly, the HIF-1 $\alpha$-dependent regulation of another hypoxiaresponsive gene, encoding the glucose transporter GLUT-1, is intact in PKC- $\beta$-null mice (34), suggesting that PKC- $\beta$ and HIF- $1 \alpha$ independently (but perhaps synergistically) modulate the transcription of oxygensensitive genes in the lung.

\section{VEGF and pulmonary stress}

The muscularization seen in precapillary pulmonary arteries is essentially identical in histological appearance whether it occurs in the chronically hypoxic rat lung or in the lung from a patient with a cardiac interatrial septum defect, a condition that leads to high pulmonary blood flow and increases shear stress but is not accompanied by hypoxia. This similarity raises the question of what common physiological response is being induced in these different settings. Clearly, the common denominator could be the presence of pulmonary vascular stress, which might be sensed by the endothelial lining and transmitted to the VSMCs. The intimate connection in vivo between endothelial cells and VSMCs is seen in their many functional interactions. For example, activation of eNOS leads to NO production, and NO relaxes the smooth muscle cells. Similarly, endothelial-cell prostacyclin acts on VSMC prostacyclin receptors. To be sure, VSMCs isolated from small pulmonary arteries can sense a reduction in the oxygenation of their environment (14) and can proliferate in culture even in the absence of endothelial cells. Nevertheless, in the hypoxic pulmonary arteriole itself, one would expect vascular endothelial cells to communicate with VSMCs. Consistent with this expectation, signaling through endothelial VEGF receptors has emerged as an important regulator of vascular remodeling $(11,12,27)$.

Chronic inhibition of VEGF-R2 with the VEGF receptor blocker SU5416 causes muscularization of arterioles in the nonhypoxic rat lung (12), suggesting that the functional interaction between VSMC and endothelial cell in precapillary pulmonary arterioles is critically altered when the endothelial cell VEGF signal transduction is perturbed. Both impaired endothelial VEGF signaling (as occurs in this rat lung model; refs. 12,35 ) and endothelial cell injury (as occurs in monocrotaline-induced pulmonary hypertension) can provoke VSMC growth (Figure 2). How the VSMC registers these changes in the endothelial cell remains uncertain, but again, endothelial $\mathrm{NO}$ and prostacyclin production, which are likely to be suppressed because of the impairment of VEGF signaling in both models, may well provide signals that control the growth of neighboring VSMCs. Another intriguing mechanism by which pulmonary stress could lead to muscularization of the vascular wall would involve the direct transdifferentiating of endothelial cells into VSMCs. This change in cellular phenotype has been observed in the culture dish (36), but it is unknown whether it can occur during vascular remodeling in hypoxic hypertension. Regardless of the nature of the stimulus or the mechanism of the response, pulmonary hypertension in these settings probably does not require vasoconstriction but can result from muscularization alone, or in association with loss of vasodilation.

\section{Endothelial-cell proliferation}

In the relatively mild forms of pulmonary hypertension described above, hyperplasia and hypertrophy are seen in the medial smooth muscle cell layer, but the pulmonary endothelium remains as a monolayer in the remodeled pulmonary arteries. In contrast, severe pulmonary hypertension, a fatal disease that ultimately leads to heart failure, is characterized by the presence of intraluminal clusters of endothelial cells $(37,38)$. We recently observed that rats treated for 3 weeks with both the VEGF-R2 blocker SU5416 and hypoxic conditions develop a severe, irreversible, and fatal form of pulmonary hypertension $(12,37)$ (Figure 3). As in patients with severe pulmonary hypertension, this process is characterized by occlusion of precapillary pulmonary arteries by clusters of proliferating endothelial cells. To account for the crucial role of chronic hypoxia in this endothelial cell growth response in the rat model, we postulate that the inhibition of the action of an endothelial cell sur-

\section{Figure 2}

Pulmonary vascular morphology of vehicle-treated (a) or SU5416-treated (b) rats exposed to Denver altitude conditions for 3 weeks. Note the increase in medial thickness in the SU5416-treated rat lungs, which extends into preacinar vessels (arrow). On the other hand, normal pulmonary arteries have well defined medial muscular layer (arrow), which becomes progressively thinner (dashed arrow) in the preacinar region.
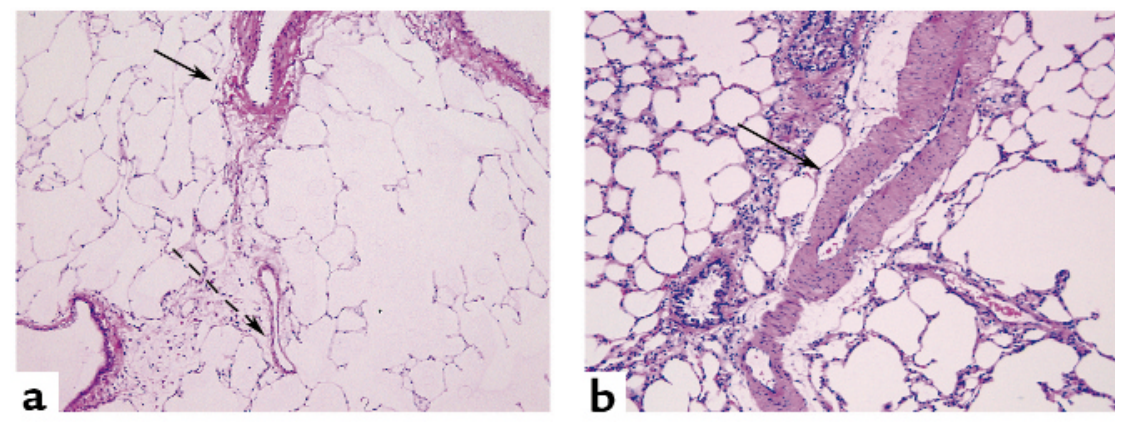

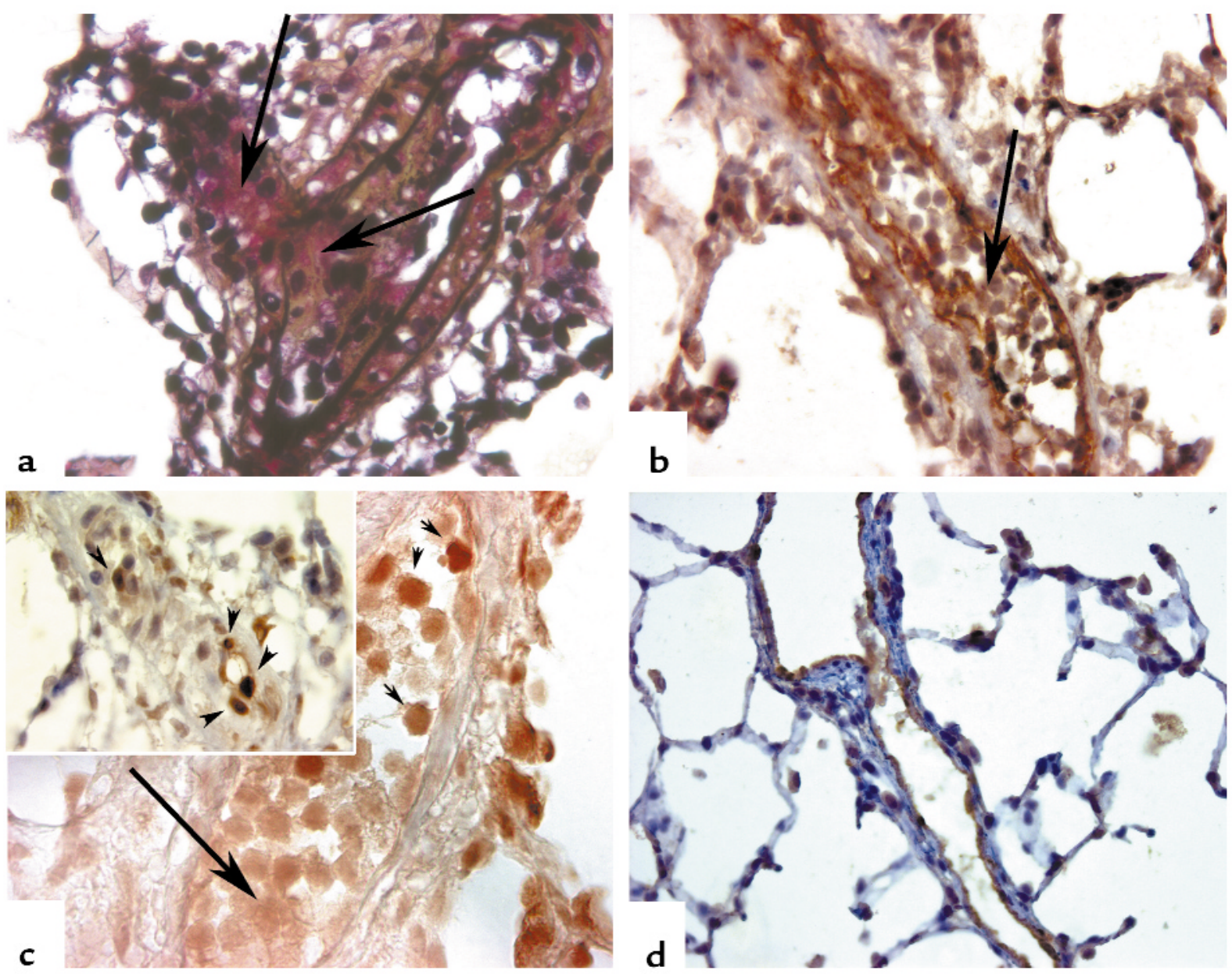

Figure 3

Lungs from chronically hypoxic rats treated with the VEGF-R2 inhibitor SU5416 for 3 weeks. (a) Intimal obliteration of precapillary pulmonary artery (arrows), resulting in almost-complete disappearance of vascular lumen; (b) the intimal obliteration is due to expansion of Factor VIII-related antigen-positive cells (arrow). (c) The intraluminal cluster of endothelial cells shows expression of the key apoptosis effector active caspase 3 (arrowhead), while endothelial cells in the center of the cluster (arrow) show evidence of proliferation with expression of proliferating cell nuclear antigen (inset, arrowheads). (d) Caspase inhibition with the broad spectrum caspase inhibitor Z-Asp-CH2, administered at the beginning of the chronic hypoxia and SU5416 treatment, results in a marked decrease in pulmonary artery pressures. The pulmonary arteries are lined by a monolayer of Factor VIII-related antigen-positive endothelial cells, with preservation of vascular lumen. (a: pentachrome staining, $\times 320$; b: Factor VIII-related antigen immunostaining, $\times 300$; c: active caspase 3 immunostaining, $\times 400$; d: Factor VIII-related antigen immunostaining, $\times 200$.)

vival factor (like VEGF) leads to apoptotic cell death of the normal endothelial cells in the pulmonary arteries of treated animals. Therefore, the treatment selects for the emergence of an apoptosis-resistant proliferating endothelial cell that cannot be killed by the VEGF-R2 blocker. Chronic hypoxia may act by increasing shear stress, especially at branching points in the affected pulmonary arteries, thus allowing the proliferation of apoptosis-resistant endothelial cells (37). In support of this model, we note that treatment with a broad-spectrum caspase inhibitor prevents the development of severe pulmonary hypertension and endothelial cell proliferation, presumably because it removes the selective pressure that otherwise acts on the endothelial cells.
By analogy to this model, human severe pulmonary hypertension may also involve the selection, within the pulmonary arterioles, of endothelial progenitors that undergo abnormal proliferation following endothelial cell injury or mutations. This model may explain the monoclonal endothelial cell growth in lungs from patients with idiopathic severe pulmonary hypertension (also known as primary pulmonary hypertension). In contrast, other conditions that lead to severe pulmonary hypertension - left-to-right heart shunts or the CREST syndrome (38) - permit polyclonal growth of endothelial cells, suggesting that the pathogenesis of these conditions does not depend on a rare somatic mutation within the endothelial cell population. We have recently found that endothelial cells in patients 
with primary pulmonary hypertension carry inactivating mutations in the genes for the type II TGF- $\beta$ receptor (TGF- $\beta$ RII) and the proapoptotic molecule Bax (M.E. Yeager et al., manuscript submitted for publication). TGF- $\beta$ RII may be a critical determinant of the balance between cell death and cell growth, and we postulate that impaired TGF- $\beta$ RII signaling could cause pulmonary endothelial cell proliferation or at least contribute to endothelial cell growth. Because these cells also exhibit instability in the structure of their microsatellite DNA repeat sequences, it is plausible that an endothelial cell mutator phenotype develops first and that mutations occur subsequently in these or other genes that control apoptosis or cell growth.

\section{From animal models to human disease?}

When we impose chronic hypoxia on animals by restricting oxygen in their air, which forms or aspects of human pulmonary hypertension are we modeling? On one view, the information we obtain is relevant, at best, only to "pure" hypoxic pulmonary hypertension, as occurs in chronic mountain disease (39) or sleep apnea syndromes. What of chronic obstructive lung diseases and interstitial lung diseases (40), which are associated not only with hypoxia/hypoxemia but also with inflammation, or the Eisenmenger syndrome, which includes high pulmonary blood flow?

Whether studies in hypoxic animals can be generalized to these more complex forms of chronic lung diseases will depend primarily on whether some final common pathway links the variety of trigger factors to precapillary muscularization. The work of Marlene Rabinovitch's group $(13,41)$, using the endothelial cell toxin monocrotaline to trigger pulmonary vascular remodeling, may point toward such a final pathway. Cowan et al. $(13,41)$ showed recently that vascular remodeling in the monocrotaline model could be blocked by inhibiting ECM turnover or by blocking the induction of the ECM protein tenascin-C. At least in this model, it appears that the composition of the ECM in and around the pulmonary vessels and the balance between secreted proteinases and proteinase inhibitors serve as important regulators of VSMC proliferation. Whether ECM metabolism plays a similar role in vascular remodeling following chronic hypoxia is not known. Another, perhaps complementary, model maintains that endothelial cell apoptosis is a critical event in pulmonary vascular remodeling (37). In principle, endothelial cell apoptosis could serve as the signal that promotes VSMC proliferation and apoptosis during vascular remodeling. Because caspases induced in apoptosis might (directly or indirectly) activate secreted proteinases, it is possible that endothelial cell apoptosis also underlies the pathological degradation of the ECM seen during development of pulmonary hypertension. Whether endothelial cell apoptosis, ECM degradation, or both together provide a common path- way to integrate signals in pulmonary vessels needs to be examined in the systems discussed above and in others that model more complex forms of lung disease.

\section{Acknowledgments}

This work has been supported by NIH grants to R.M. Tuder (1RO1 HL-60195-01) and N.F. Voelkel (1RO1 HL-60913-01) and by the Shirley Kiner Witham Memorial Pulmonary Hypertension Research Fund.

1. Will, D.H., Alexander, A.F., Reeves, J.T., and Grover, R.F. 1962. High altitude-induced pulmonary hypertension in normal cattle. Circ. Res. 10:172-177.

2. Voelkel, N.F., Wiegers, U., Sill, V., and Trautmann, J. 1977. Kinetic study on the lung DNA-synthesis stimulated by chronic high altitude hypoxia. Thorax. 32:578-584.

3. Rabinovitch, M., Gamble, W., Nadas, A.S., Miettinen, O., and Reid, L. 1979. Rat pulmonary circulation after chronic hypoxia: hemodynamic and structural features. Am. J. Physiol. 236:H818-H827.

4. Stenmark, K., et al. 1987. Severe pulmonary hypertension and arterial adventitial changes in newborn calves at $4300 \mathrm{~m}$. J. Appl. Physiol. 62:821-830.

5. Voelkel, N.F., Tuder, R.M., Lepley, R.A., and Fitzpatrick, F.A. 1996. Inhibition of 5-lipoxygenase-activating protein (FLAP) reduces pulmonary vascular reactivity and pulmonary hypertension in hypoxic rats. J. Clin. Invest. 97:2491-2498.

6. Li, H., et al. 1994. Enhanced endothelin-1 and endothelin receptor gene expression in chronic hypoxia. J. Appl. Physiol. 77:1451-1459.

7. Eddahibi, S., et al. 2000. Attenuated hypoxic pulmonary hypertension in mice lacking the 5-hydroxytryptamine transporter gene. J. Clin. Invest. 105:1555-1562.

8. Isaacson, T.C., Hampl, V., Weir, E.K., Nelson, D.P., and Archer, S.L. 1994. Increased endothelium-derived NO in hypertensive pulmonary circulation of chronically hypoxic rats. J. Appl. Physiol. 76:933-940.

9. Fagan, K.A., et al. 1999. The pulmonary circulation of homozygous or heterozygous eNOS-null mice is hyperresponsive to mild hypoxia. J. Clin. Invest. 103:291-299.

10. Geraci, M.W., et al. 1999. Selective pulmonary prostacyclin synthase overexpression in transgenic mice protects against the development of hypoxic pulmonary hypertension. J. Clin. Invest. 103:1509-1515.

11. Tuder, R.M., Flook, B., and Voelkel, N.F. 1995. Increased gene expression for VEGF and VEGF receptors KDR/Flk and Flt in lungs of chronically hypoxic rats. J. Clin. Invest. 95:1798-1807.

12. Tuder, R.M., Taraseviciene, L., Hirth, P., and Voelkel, N.F. 1999. Inhibition of the VEGF receptor causes severe pulmonary hypertension (PAH) in rats. Circulation. 100:I241. (Abstr.)

13. Cowan, K.N., et al. 2000. Complete reversal of fatal pulmonary hypertension in rats by a serine elastase inhibitor. Nat. Med. 6:698-702.

14. Yuan, X.J., Goldman, W.F., Tod, M.L., Rubin, L.J., and Blaustein, M.P. 1993. Hypoxia reduces potassium currents in cultured rat pulmonary but not mesenteric arterial myocytes. Am. J. Physiol. Lung Cell. Mol. Physiol. 264:L116-L123.

15. Sato, K., Morio, Y., Morris, K.G., Rodman, D.M., and McMurtry, I.M. 2000. Mechanism of hypoxic pulmonary vasoconstriction involves $\mathrm{ET}_{\mathrm{A}}$ receptor-mediated inhibition of $\mathrm{K}_{\mathrm{ATP}}$ channel. Am. J. Physiol. Lung Cell. Mol. Physiol. 278:L434-L442.

16. Rodman, D.M., and Voelkel, N.F. 1997. Regulation of vascular tone. In The lung: scientific contribution. R.G. Crystal, J.B. West, P.J. Barnes, N.S. Cherniack, and E.R. Weibel, editors. Raven Press. New York, New York, USA. 1473-1492.

17. Semenza, G.L., and Wang, G.L. 1992. A nuclear factor induced by hypoxia via de nova protein synthesis binds to the human erythropoietin gene enhancer at a site required for transcriptional activation. Mol. Cell Biol. 12:5447-5454.

18. Wang, G.L., Jiang, B.H., Rue, E.A., and Semenza, G.L. 1995. Hypoxiainducible factor 1 is a basic-helix-loop-helix-PAS heterodimer regulated by cellular O2 tension. Proc. Natl. Acad. Sci. USA. 92:5510-5514.

19. Forsythe, J.A., et al. 1996. Activation of vascular endothelial growth factor gene transcription by hypoxia-inducible factor 1. Mol. Cell Biol. 16:4604-4613.

20. Isner, J. 2000. Tissue responses to ischemia: local and remote responses for preserving perfusion of ischemic muscle. J. Clin. Invest. 
106:615-619.

21. Semenza, G.L. 2000. Surviving ischemia: adaptive responses mediated by hypoxia-inducible factor $1 . \mathrm{J}$. Clin. Invest. In press.

22. Barazzone, C., and White, C.W. 2000. Mechanisms of cell injury and death in hyperoxia: role of cytokines and Bcl-2 family proteins. Am. J. Respir. Cell Mol. Biol. 22:517-519.

23. Tuder, R.M., and Voelkel, N.F. 1998. Pulmonary hypertension and inflammation. J. Lab. Clin. Med. 132:16-24.

24. Demiryurek, A.T., and Wadsworth, R.M. 1999. Superoxide in the pulmonary circulation. Pharmacol. Ther. 84:355-365.

25. Stanbrook, H., Morris, K.G., and McMurtry, I.F. 1984. Prevention and reversal of hypoxic pulmonary hypertension by calcium antagonists. Am. Rev. Respir. Dis. 130:81-85.

26. Ono, S., Westcott, J.Y., and Voelkel, N.F. 1992. PAF-antagonists inhibit pulmonary vascular remodeling induced by hypobaric hypoxia in rats. J. Appl. Physiol. 73:1084-1092.

27. Tuder, R.M., Allard, J., and Voelkel, N.F. 1996. Role of vascular endothelial growth factor in hypoxia and monocrotaline induced pulmonary hypertension. Circulation. 94:164. (Abstr.)

28. Wheeler-Jones, C., et al. 1997. Vascular endothelial growth factor stimulates prostacyclin production and activation of cytosolic phospholipase $\mathrm{A}_{2}$ in endothelial cells via $\mathrm{p} 42 / \mathrm{p} 44$ mitogen-activated protein kinase. FEBS Lett. 420:28-32.

29. Shen, B.Q., Lee, D.Y., and Zioncheck, T.F. 1999. Vascular endothelial growth factor governs endothelial nitric-oxide synthase expression via a KDR/Flk-1 receptor and a protein kinase C signaling pathway. J. Biol. Chem. 274:33057-33063.

30. Yu, A.Y., et al. 1999. Impaired physiological responses to chronic hypoxia in mice partially deficient for hypoxia-inducible factor $1 \alpha$.J. Clin. Invest. 103:691-696.

31. Iyer, N.V., et al. 1998. Cellular and developmental control of O2 homeostasis by hypoxic-inducible factor $1 \alpha$. Genes Dev. 12:149-162.

32. Cott, G.R., Westcott, J.Y., and Voelkel, N.F. 1990. Synthesis of eicosanoids by rat alveolar type II cells in vitro. Am. J. Physiol. Lung Cell. Mol. Physiol. 2:L179-L187.

33. Yan, S.H., et al. 1998. Tissue factor transcription driven by Egr-1 is a critical mechanism of murine pulmonary fibrin deposition in hypoxia. Proc. Natl. Acad. Sci. USA. 95:8298-8303.

34. Yan, S.F., et al. 2000. Protein kinase C- $\beta$ and oxygen deprivation: a novel Egr-1-dependent pathway for fibrin deposition in hypoxemic vasculature. J. Biol. Chem. 275:11921-11928.

35. Alon, T., et al. 1995. Vascular endothelial growth factor acts as a survival factor for newly formed retinal vessels and has implications for retinopathy of prematurity. Nat. Med. 1:1024-1028.

36. Arciniegas, E., Sutton, A.B., Alled, T.D., and Schor, A.M. 1992. Transforming growth factor beta-1 promoted the differentiation of endothelial cells into smooth muscle-like cells in vitro. J. Cell Sci. 103:521-529.

37. Taraseviciene-Stewart, L., et al. 2000. Inhibition of the VEGF receptor-2 combined with chronic hypoxia causes cell death-dependent pulmonary endothelial cell proliferation and severe pulmonary hypertension. FASEB J. In press.

38. Lee, S.D., et al. 1998. Monoclonal endothelial cell proliferation is present in primary but not secondary pulmonary hypertension. J. Clin. Invest. 101:927-934.

39. Arias-Stella, J., and Saldana, M. 1963. Terminal portion of the pulmonary arterial in people native to high altitudes. Circulation. 28:915-925.

40. Tuder, R.M., Cool, C., Jennings, C., and Voelkel, N.F. 1998. Pulmonary vascular involvement in interstitial lung disease. In Interstitial lung disease. 3rd edition. M.I. Schwarz and T.E. King, editors. B.C. Decker. Hamilton, Ontario, Canada. 251-263.

41. Cowan, K.N., Jones, P.L., and Rabinovitch, M. 2000. Elastase and matrix metalloproteinase inhibitors induce regression and tenascin$\mathrm{C}$ antisense prevents progressive vascular disease. J. Clin. Invest. 105:21-34. 